\title{
Palifermin for management of treatment-induced oral mucositis in cancer patients
}

\author{
Andrei Barasch' \\ Joel Epstein ${ }^{2}$ \\ Ken Tilashalski' \\ 'Department of Diagnostic Sciences, \\ University of Alabama at Birmingham, \\ Birmingham, AL, USA; ${ }^{2}$ Department \\ of Oral Medicine and Diagnostic \\ Sciences and Otolaryngology/Head \\ and Neck Surgery, University of \\ Illinois at Chicago, Chicago, IL, USA
}

\begin{abstract}
Oral mucositis (OM) remains a major side effect of various cancer therapies, which exacts a significant price in terms of morbidity and cost of care. Efforts aimed at prevention and/or therapy of OM have been largely unsuccessful. Few agents have shown efficacy, and even those were applicable to limited types of patients. The advent of small-molecule targeted agents opened new possibilities for intervention in the mucopathogenic processes induced by cancer therapies. One of these agents, recombinant human keratinocyte growth factor (KGF), has been studied extensively and has shown promising results in reducing chemotherapy induced OM. This drug's effects on stem cell engraftment, graft-versus-host disease and other treatment-induced morbidities remain undefined. In this article we evaluate the pre-clinical and clinical evidence and discuss the clinical applications of KGF as an adjunct therapeutic agent in oncology.

Keywords: mucositis, cancer, therapy, palifermin
\end{abstract}

\section{Introduction}

In spite of the recent introduction of targeted therapies for malignant diseases, the vast majority of antineoplastic agents have a relatively non-specific cytotoxic mode of action. Consequently, cancer treatments continue to be characterized by high morbidity and mortality. By virtue of their active metabolic and mitotic processes, some of the most commonly affected "innocent bystanders" are the hematopoietic and gastrointestinal mucosal cells. In the 1990s we witnessed significant advances in prophylaxis and therapy of hematopoietic side effects of toxic cancer drugs through the discovery and introduction to market of specific immune cell colony growth factors. ${ }^{1}$ These advances led to an effective decrease in severity and duration of immune cytopenias and their associated infectious morbidity. An additional consequence was the shift in importance of the mucosal side effects, which have now become the limiting toxicity in various cancer regimens. Until recently, gastrointestinal mucositis treatment has been considered an unmet need, with most sufferers receiving only palliative agents and nutritional support. ${ }^{1-3}$

The process of aero-digestive tract injury by cytotoxic therapies remains incompletely elucidated. The most recent and compelling theory was proposed by Sonis in $2004^{2}$ and is based on observational phenomena in humans and experimental evidence in a hamster animal model. This theory contends that the injurious process begins with damage to the vasculature and connective tissues underlying the mucosa, which is followed by local signal amplification and release of pro-inflammatory cytokines. Resultant vascular leakage exacerbates the inflammation and leads to epithelial destruction followed by bacterial colonization of sub-epithelial tissues. As the cytotoxic effects wane with drug discontinuation, the wound healing process predominates and restores epithelial integrity, albeit a full return to tissue health requires significantly more time.

Oral mucositis $(\mathrm{OM})$ is relatively common in cancer patients and ranges in incidence from close to $100 \%$ in some bone marrow transplantation (BMT) myeloablative regimens and radiotherapy for head and neck cancer to less than $10 \%$ in solid tumors
Correspondence: Dr Andrei Barasch University of Alabama at Birmingham, SDBI I I, I 530 3rd Avenue S, Birmingham, AL 35294-0007, USA

Tel +l 2059964418

Fax + I 2959750603

Email abarasch@uab.edu 
treated with milder regimens or targeted protocols. ${ }^{3}$ The morbidity of mucositis in affected patients is significant and occurrence of moderate-severe OM has been associated with increases in the number of hospital days, parenteral nutrition, need for narcotic-based parenteral analgesia and mortality. ${ }^{4}$ The monetary cost of OM has been estimated at over US\$40,000 per patient afflicted by ulcerative lesions, based on Medicare reimbursement rates. Thus, therapyinduced OM is far from trivial and recent efforts have been directed at finding prophylactic and therapeutic measures. To date, relatively few compelling results have been achieved, chief among those being the introduction and testing of the recombinant keratinocyte growth factor (KGF) palifermin. We will now discuss this medication and the evidence of its efficacy presented in the literature.

\section{Preclinical studies}

$\mathrm{KGF}$ is an epithelial cell growth factor expressed by fibroblasts and endothelial cells, typically in response to proinflammatory cytokines and hormones. ${ }^{5,6}$ This cytokine plays an important role in regulating epithelial-mesenchymal interactions and in maintaining epithelial integrity. ${ }^{7}$ Preclinical studies have described increased proliferation and decreased apoptosis of epithelial cells in response to KGF administration. Enhanced neovascularization and collagen deposition were also noted in a wound healing animal model. ${ }^{8}$ Generally, wound healing in this model was consistently enhanced by either topical or systemic KGF and intestinal anastomoses healed faster and withstood more tensile force and skin wounds resolved in significantly less time.

The drug palifermin (recombinant human DeltaN23KGF, Biovitrum AB Stockholm, Sweden) has gained scientific attention in late 1990s with the publication of animal study results indicating efficacy in protection of oral and intestinal epithelia from negative effects of radiation and chemotherapy..$^{9,10}$ Initial results described protection of the gastrointestinal mucosa in mice treated with lethal doses of radiation or chemotherapy. ${ }^{9}$ Animals exposed to KGF had lower mortality that was attributed to the mucosal cytoprotection. Increasing the optimism, other studies published at the same time indicated that KGF had no protective effect on human squamous cell carcinoma cell lines exposed to ionizing radiation in vitro and in vivo. ${ }^{11}$

Farrell et al then studied the effect of palifermin on mouth mucosa in mice exposed to ionizing radiation and described a reversal of the radiation-induced inhibition of cell growth in irradiated animals. ${ }^{12}$ Further, Potten et al studied the effect of KGF on mouse tongue mucosa and similarly noted a significant increase in cell proliferation. When animals were subsequently exposed to high dose radiation, both incidence and duration of ulceration were reduced. ${ }^{13}$ Dorr and co-workers ${ }^{14}$ also exposed mice to radiation and observed the effects of KGF applied before, during or after exposure. A significant reduction in incidence of radiationinduced ulceration was noted for all three protocols. The positive effect was most pronounced when KGF was given after radiation, suggesting that the mechanism responsible for benefits was increase in tissue repair. However, in a subsequent report, ${ }^{15}$ the same team found longer lasting ulceration when KGF was administered after the onset of ulceration as compared to control irradiated animals. The reason for this apparent contradiction has not been elucidated but the authors recommended that $\mathrm{KGF}$ be administered prior to appearance of ulcerative mucositis.

Expanding the area of study, other researchers ${ }^{16}$ demonstrated milder forms of graft-vs-host disease (GVHD) after chemoradiotherapy conditioning followed by allogeneic bone marrow transplantation (BMT) in animals pretreated with palifermin. These animals also had enhanced engraftment, presumably by mechanisms independent of the repair of cytotoxic-induced tissue injury. One possible explanation was presented by Seggewiss et $\mathrm{al}^{17}$ who described improved immune reconstitution and thymus preservation in monkeys treated with myeloablative radiation and KGF. Finally, a group studied the effect of radiation on salivary gland cells and saliva production in mice that received palifermin before and/or after exposure to the cytotoxic therapy. ${ }^{18}$ The authors concluded that KGF alleviated radiation-induced damage to the glands by stimulating growth of the acinar progenitor stem cell pool. While radiation sensitivity was not affected, the increase in the number of stem cells compensated for radiation-induced killing. Gland function measured in vivo was also improved.

These animal studies were in accord that palifermin has a potential role as a disease modifier in radiation-induced mucosal injury, immune reconstitution and in GVHD. However, other studies created serious doubt about potential use of this cytokine for chemotherapy-induced damage. ${ }^{19-21}$ A group in Oklahoma ${ }^{19}$ reported a dose-dependent KGF induction of estrogen receptor-positive breast cancer cell proliferation and motility, even at very low drug levels. Contrary to previous suggestions, these authors concluded that KGF inhibition may provide therapeutic benefits for treatment of metastatic breast cancer. ${ }^{20} \mathrm{~A}$ similar study in human endometrial carcinoma cells also showed enhanced growth as a result of KGF addition. ${ }^{21}$ 
Similar worries were expressed in a recent article, which described KGF-induced resistance of cancer cells to specific chemotherapeutic agents. ${ }^{22}$ The authors reported complete inhibition of breast cancer and normal epithelial cell killing by 5 -florouracil (5-FU) when KGF was added to cell culture. Further, the inhibitory effect of tamoxifen on breast cancer cells was also abrogated by the addition of the cytokine. In contrast, another recent study ${ }^{23}$ tested the effect of combining palifermin with various cytotoxic and biologic medications on human head and neck and colorectal cancer xenografts in mice. Medications tested included 5-FU, cisplatin and three targeted agents. This study showed that palifermin neither influenced tumor growth, nor interfered with the cell killing activity of any of the tested drugs. Further, a report by Gibson et al ${ }^{24}$ showed that KGF administered to breast cancer-bearing rats before treatment with methotrexate actually enhanced drug-induced apoptosis in both normal intestinal and malignant cells. Thus, no protection of mucosal tissues was observed but there was a beneficial effect on the tumor.

An explanation for these contradictory results is not readily apparent. While most experiments that showed enhanced tumor growth and inhibition of cytotoxic properties of drugs were performed with breast cancer cells, ${ }^{19-22}$ the malignant lines used in the studies that found no interference ${ }^{12,23}$ were of squamous cell and colorectal origins. Nevertheless, all tested cell lines were KGF-receptor positive, which implies that their behavior should be similar. Further, the former studies were performed primarily in cell culture while the latter were animal trials. This suggests that effects of KGF in vitro may not translate to in vivo results, where other biological processes may account for a difference in behavior. The odd results reported by Gibson et $\mathrm{a}^{24}$ may be due to the use of methotrexate, which is an antimetabolite with unique characteristics.

\section{Clinical evidence}

\section{Radiotherapy}

The initial introduction of palifermin to human trials occurred in a phase I/II dose-escalation experiment in head and neck cancer patients receiving chemoradiation. ${ }^{25}$ Chemotherapy-naïve patients were treated with 5-FU and cisplatin concomitantly with radiotherapy. KGF was administered intravenously $(20,40,60 \mathrm{or} 80 \mu \mathrm{g} / \mathrm{kg})$ to a minimum of 12 patients per dose. Results from 60 randomized patients (46 palifermin, 14 placebo) showed that the drug was tolerated relatively well even at the highest dose, with minimal side effects consisting mostly of skin flushing, temporary elevation of serum lipase and amylase and hypersalivation. Duration of severe mucositis and salivary gland toxicity were significantly reduced in the patients treated with palifermin.

The same group reported the results of a phase II study of palifermin in a similar group of patients. ${ }^{26}$ Randomly assigned 99 head and neck cancer patients (2:1 palifermin $60 \mu \mathrm{g} / \mathrm{kg}$ or placebo once weekly) were assessed for duration of moderate-severe oral mucositis. Results were not statistically different for the whole group. However, subgroup analyses showed that duration of mucositis was significantly reduced in those patients who received hyperfractionated radiation and palifermin; conversely, duration of mucositis was increased in those treated with standard dose radiation and the cytokine. Adverse events, tumor response and overall survival were similar for the two groups. It is interesting that patients treated with hyperfractionated radiation had benefit, as this delivery of radiation is associated with increased acute mucosal toxicity. The results of this post-hoc analysis are not easily explained and the authors justly conclude that additional studies are necessary to determine the efficacy of palifermin for chemoradiation-induced mucositis. A phase III trial of palifermin in head and neck cancer patients has recently completed accrual but results are not yet available.

\section{Chemotherapy}

A second phase I study was undertaken in colorectal cancer patients treated with a 5-FU-based chemotherapy protocol. ${ }^{27}$ This was a randomized (2:1) study of 81 patients who received palifermin $(1,10,20,40,60$ or $80 \mu \mathrm{g} / \mathrm{kg} /$ day $)$ or placebo intravenously on days 4 to 8 . Unlike the previous clinical study, dose-limiting toxicities were found at 60 and $80 \mu \mathrm{g} / \mathrm{kg}$. Incidence of severe mucositis was decreased in palifermin patients, albeit it did not reach statistical significance $(p=0.6)$. We note that the study was not powered to show significant mucositis effects and the dose of $1 \mu \mathrm{g} / \mathrm{kg}$ may have been insufficient to produce any effects on mucositis.

A phase II trial was reported only in abstract form. ${ }^{28}$ This study established the palifermin dose at $60 \mu \mathrm{g} / \mathrm{kg}$ injected intravenously for 3 days before conditioning and 3 days after marrow transplantation in BMT patients. The practice-changing study was a phase III multicenter clinical trial that compared oral mucositis in 106 palifermin$(60 \mu \mathrm{g} / \mathrm{kg} /$ day for 3 consecutive days before initiation of cytotoxic therapy and on days 0,1 , and 2 after transplantation) vs 106 placebo-treated autologous BMT patients who received marrow-ablative chemotherapy for hematologic malignancies. ${ }^{29}$ Severe mucositis was reported in $63 \%$ of 
the patients in the palifermin group and $98 \%$ of the patients treated with placebo $(\mathrm{p}<0.001)$. Duration of mucositis was also reduced by an average of 3 days in the treatment group. Incidence of WHO grade IV mucositis was $21 \%$ with palifermin compared to $62 \%$ with placebo $(\mathrm{p}<0.001)$. Patient-reported symptoms, swallowing, use of narcotic analgesia and use of parenteral nutrition coincided with the mucositis curve and were significantly reduced in the palifermin-treated patients. General well-being was also improved in the treatment group. Reported adverse effects were mild to moderate and consisted mostly of skin effects, cough and taste alterations. Transient increases in serum amylase and lipase were also common but values returned to normal soon after treatment discontinuation. Short-term disease-free survival was similar in the two groups but long-term effects have not been reported. This study was funded by the palifermin's manufacturer and most authors had received financial benefits from the company. Nevertheless, the results clearly showed the efficacy of the drug in reducing therapy-induced mucositis and improving shortterm outcomes in chemoradiotherapy-treated hematologic cancer patients.

A similar study published 2 years later $^{30}$ confirmed the benefits of palifermin on oral mucositis in BMT populations. This was an open-label cohort trial of the drug where patients were compared to historic controls and most results paralleled those of the Spilberger study. ${ }^{29}$ However, no benefits were found in use of narcotic medication, dietary intake, general well-being or time to engraftment for the KGF-treated 32 patients. This study also did not report long-term follow-up or effects of palifermin on GVHD.

Other cancer patient groups have been studied. A report by Rosen et al $^{31}$ described the effects of palifermin in metastatic colorectal cancer patients treated with 5-FU and leukovorin. Sixty-four patients were randomly assigned to KGF or placebo. The authors reported a decrease in incidence of severe oral mucositis with palifermin but no effect on diarrhea. Interestingly, despite the possible interference of KGF with the efficacy of 5-FU, the overall 2-year survival was similar in the two groups. Longer-term survival was not reported. Studying a cohort of 10 patients with previous high-dose methotrexate-induced severe oral mucositis, Schmidt et al ${ }^{32}$ also reported significant reductions in incidence and duration of severe lesions when KGF was administered in subsequent cycles. In this group of patients, the incidence of infection was also substantially lower with palifermin treatment. These preliminary studies show that KGF can be efficacious even outside the BMT setting. However, longer follow-up and rigorous methodology must be employed in future studies to insure that disease recurrence and long-term survival are not negatively affected by palifermin.

Results from Spielberger et al study ${ }^{29}$ were used post-hoc to calculate the economic consequences of palifermin in BMT patients. ${ }^{33}$ This analysis was based upon estimated Medicare reimbursement and concluded that the price of palifermin was easily offset by savings from reduced hospital stay and palliative medication. In final analysis, an average non-statistically significant saving of US\$3,595 per patient was achieved by using the drug.

\section{GVHD}

Following the positive results in animal studies,,${ }^{16}$ the effect of KGF on GVHD became a topic of interest. Three recent studies address this issue. Langner et $\mathrm{al}^{34}$ reported the results of a muticenter study that enrolled 30 allogeneic BMT patients to receive palifermin and compared them to matched historic controls. Consistent with previous studies, incidence, severity and duration of oral mucositis as well as use of narcotic medication and parenteral nutrition were significantly reduced in the palifermin group. However, no benefits were seen for the incidence of acute GVHD, febrile neutropenia, infectious episodes or hematopoietic recovery. Short-term survival was similarly not affected. The second study was a randomized trial ${ }^{35}$ that used higher doses of palifermin $(240-720 \mu \mathrm{g} / \mathrm{kg})$ in 100 allogeneic BMT patients. Similar to the Langner study, the authors reported beneficial effects for oral mucositis but not on acute GVHD, engraftment or survival. Unlike the previous reports, these authors provided long-term follow up data, which showed no differences in incidence of infection, chronic GVHD or long-term survival. Lastly, the results of a multicenter study ${ }^{36}$ on 53 BMT Polish patients compared to 53 matched historic controls confirmed the positive effects of palifermin on severity and duration of mucositis but also claimed a significant reduction in prevalence of acute GVHD. This study followed the exact protocol described by Spielberger et al. ${ }^{29}$

The former two studies appear to dim the hope that palifermin may be efficacious in reducing GVHD and incidence of infection, or provide any benefit to engraftment, while the third keeps the flame alive. Nevertheless, all studies confirm the positive effects of palifermin on oral mucositis. Discrepancies in results for use of analgesic medication and parenteral nutrition will have to be settled in future studies. 


\section{Similar medications}

The positive results obtained with palifermin encouraged investigation of other possible mucositis modulators from the same cytokine family. KGF-2 (repifermin) was the first to be studied. In vitro results affirmed the lack of effect of the drug on 30 carcinoma cell lines, while animal experiments confirmed no tumor growth activity. ${ }^{37} \mathrm{~A}$ phase I/II trial that followed also reported encouraging results, with significant reduction of mucositis and no significant side effects for repifermin-treated patients. ${ }^{38}$ Nevertheless, in February 2004 the manufacturer announced the abandonment of repifermin after an unpublished phase II study did not meet its primary end point.

Recombinant fibroblast growth factor (FGF)-20 (velafermin) is another signaling molecule that may be beneficial to mucosal cytoprotection. In an hamster model for therapy-induced mucositis, administration of intraperitoneal velafermin reduced both duration and severity of mucosal lesions. ${ }^{39,40}$ In a phase I open-label human study of FGF-20 in 30 autologous BMT patients, ${ }^{41}$ the authors reported no serious adverse effects. Nevertheless, this drug too was dropped in October 2007 when the company reported that FGF-20 failed to meet its primary endpoint in the management of mucositis.

\section{Conclusions}

Until recently, cancer therapy-induced oral mucositis was considered an unmet need of the oncology patient population. With the introduction of palifermin, this paradigm appears to have changed, at least for hematopoietic stem cell transplant patients treated with high-dose cytotoxic regimens, and perhaps for other cancer patients. Study results from well-designed multicenter clinical trials leave little doubt that KGF reduces incidence, duration and severity of mucosal lesions in the oral cavity. However, whether or not this effect translates into a similarly reduced incidence of infection, febrile neutropenia and improved nutrition and survival is not clear. Similarly, the GVHD benefits observed in animal models are not apparent in human studies.

Despite results from two small studies..$^{31,32}$ the question of equivalent efficacy of palifermin in other cancer populations, particularly in those with solid tumors, remains open. The reports of stimulated growth and mobility of various cancer cell lines by KGF, as well as its potential inhibition of cytotoxicity of 5-FU and tamoxifen mandate further investigation. Patients with breast cancer as well as those treated with regimens containing 5-FU or tamoxifen must be approached very cautiously.

\section{Disclosures}

None of the authors have conflicts of interest to disclose.

\section{References}

1. Raber-Durlacher JE, Barasch A, Peterson DE, et al. Oral complications and management considerations in patients treated with high-dose cancer chemotherapy. Support Cancer Ther. 2004;4:219-230.

2. Sonis ST. The pathobiology of mucositis. Nat Rev Cancer. 2004;4:277-284.

3. Barasch A, Peterson DE. Risk factors for ulcerative oral mucositis in cancer patients: unanswered questions. Oral Oncol. 2003;39:91-100.

4. Sonis ST, Oster G, Fuchs H, et al. Oral mucositis and the clinical and economic outcomes of hematopoietic stem-cell transplantation. $J \mathrm{Cli}$ Oncol. 2001;19:2201-2205.

5. Rubin JS, Bottaro DP, Chedid M, et al. Keratinocyte growth factor Cell Biol Int. 1995; 19:399-411.

6. MacDonald KP, Hill GR. Keratinocyte growth factor (KGF) in hematology and oncology. Curr Pharm Des. 2002;8:395-403.

7. McKeown ST, Hyland PL, Locke M, Mackenzie IC, Irwin CR. Keratinocyte growth factor and scatter factor expression by regionally defined oral fibroblasts. Eur J Oral Sci. 2003;111:42-50.

8. Jeschke MG, Richter G, Hofstadter F, Herndon DN, Perez-Polo JR, Jauch KW. Non-viral liposomal Keratinocyte growth factor (KGF) cDNA gene transfer improves dermal and epidermal regeneration through stimulation of epithelial and mesenchymal factors. Gene Ther. 2002;9:1065-1074.

9. Farrell CL, Bready JV, Rex KL, et al. Keratinocyte growth factor protects mice from chemotherapy and radiation-induced gastrointestinal injury and mortality. Cancer Res. 1998;58:933-939.

10. Farrell CL, Rex KL Chen JN, et al. The effects of keratinocyte growth factor inpreclinical models of mucositis. Cell Prolif. 2002; 35 Suppl 1:78-85.

11. Ning S, Shui C, Khan WB, et al. effects of keratinocyte growth factor on the proliferation and radiation survival of human squamous cell carcinoma cell lines in vitro and in vivo. Int J Rad Oncol Biol Phys. 1998;40:177-187.

12. Farrell CL, Rex KL, Kaufman SA, et al. Effects of keratinocyte growth factor in the squamous epithelium of the upper aerodigestive tract of normal and irradiated mice. Int J Radiat Biol. 1999;75:609-620.

13. Potten CS, Booth D, Cragg NJ, et al. Cell kinetic studies in the murine ventral tongue epithelium: mucositis induced by radiation and its protection by pretreatment with keratinocyte growth factor (KGF). Cell Prolif. 2002;35 (suppl 1):32-47.

14. Dorr W, Noack R, Spekl K, Farrell CL. Modification of oral mucositis by keratinocyte growth factor: single radiation exposure. Int J Radiat Biol. 2001;77:341-347.

15. Dorr W, Spekl K, Farrell CL. The effect of keratinocyte growth factor on healing of manifest radiation ulcers in mouse tongue epithelium. Cell Prolif. 2002;35 (suppl 1):86-92.

16. Panoskaltsis-Mortari A, Taylor PA, Rubin JS, et al. Keratinocyte growth factor facilitates alloengraftment and ameliorates graft-versus-host disease in mice by a mechanism independent of repair of conditioning-induced tissue injury. Blood. 2000;96:4350-4356.

17. Seggwiss R, Lore K, Guenaga FJ, et al. Keratinocyte growth factor auguments immune reconstitution after autologous hematopoietic progenitor cell transplantation in rhesus monkeys. Blood. 2007;110:441-449.

18. Lombaert IM, Brunsting JF, Wierenga PK, Kampinga HH, de Haan G, Coppes RP. Keratinocyte growth factor prevents radiationdamage to salivary glands by expansion of the stem/progenitor pool. Stem Cells. 2008;26:2595-2601.

19. Nguyen TN, Zang XP, Pento JT. Keratinocyte growth factor stimulates the migration and proliferation of breast cancer cells in a culture wounding model. Pharmacol Res. 2002;46:179-183.

20. Zang XP, Nguyen TN, Pento JT. Specific and non-specific KGF-induced breast cancer cell motility. Anticancer Res. 2002;22:2539-2545. 
21. Taniguchi F, Harada T, Sakamoto Y, et al. Activation of mitogen-activated protein kinase pathway by keratinocyte growth factor or fibroblast growth factor-10 promotes cell proliferation in human endometrial carcinoma cells. J Clin Endocrinol Metab. 2003;88:773-780.

22. Rotolo S, Ceccarelli S, Romano F, Frati L, Marchese C, Angeloni A. Silencing of keratinocyte growth factor receptor restores 5-fluorouracil and tamoxifen efficacy in responsive cancer cells. PLOS ONE. 2008; 3:e2528.

23. Brake R, Starnes C, Lu J, et al. effects of palifermin on antitumor activity of chemotherapeutic and biological agents in human head and neck and colorectal carcinoma xenograft models. Mol Cancer Res. 2008;6:1337-1346.

24. Gibson RJ, Keefe DM, Clarke JM, et al. The effect of keratinocyte growth factor on tumor growth and small intestinal mucositis after chemotherapy in the rat with breast cancer. Cancer Chemother Pharmacol. 2002;50:53-58.

25. Brizel DM, Herman T, Goffinet D, et al. A phase I/II trial of escalating doses of recombinant human keratinocyte growth factor (rHuKGF) in head and neck cancer (HNC) patients receiving radiotherapy (RT) with concurrent chemotherapy (CCT). (Abstr). Int J Radiat Oncol Biol Phys. 2001;51 suppl 1:40.

26. Brizel DM, Murphy BA, Rosenthal DI, et al. Phase II study of palifermin and concurrent chemoradiation in head and neck squamous cell carcinoma. J Clin Oncol. 2008;26:2489-2496.

27. Meropol NJ, somer RA, Gutheil J, et al. Randomized phase I trial of recombinant human keratinocyte growth factor plus chemotherapy: potential role as mucosal protectant. J Clin Oncol. 2003;21:1452-1458.

28. Spielberger RT, Stiff P, Emmanouilides C, et al. Efficacy of recombinant human keratinocyte growth factor (rHuKGF) in reducing mucositis in patients with hematologic malignancies undergoing autologous peripheral blood progenitor cell transplantation (auto-PBPCT) after radiation-based conditioning: results of a phase 2 trial [Abstract]. Proc Am Soc Clin Oncol. 2001;20:7a.

29. Spielberger R, Stiff P, Bensinger W, et al. Palifermin for oral mucositis after intensive therapy for hematologic cancers. $N$ Engl J Med. 2004;351:2590-2598.

30. Horsley P, Bauer JD, Mazkowiack R, Gardner R, Bashford J. Palifermin improves severe mucositis, swallowing problems, nutrition impact symptoms, and length of stay in patients undergoing hematopoietic stem cell transplantation. Support Care Cancer. 2007;15:105-109.
31. Rosen LS, Abdi E, Davis ID, et al. Palifermin reduces the incidence of oral mucositis in patients with metastatic colorectal cancer treated with fluorouracil-based chemotherapy. J Clin Oncol. 2006;24:5194-5200.

32. Schmidt E, Thoennissen NH, Rudat A, et al. Use of palifermin for the prevention of high-dose methotrexate-induced oral mucositis. Ann Oncol. 2008;19:1644-1649.

33. Elting LS, Shih YC, Stiff PJ, et al. Economic impact of palifermin on the cost of hospitalization for autologous hematopoietic stem-cell transplant: analysis of phase 3 trial results. Biol Blood Marrow Transplant. 2007; 13:806-813.

34. Langner S, Staber P, Schub N, et al. Palifermin reduces incidence and severity of oral mucositis in allogeneic stem-cell transplant recipients. Bone Marrow Transplant. 2008;42:275-279.

35. Levine JE, Blazar BR, DeFor T, Ferrara JL, Weisdorf DJ. Long-term follow-up of a phase I/II randomized, placebo-controlled trial of palifermin to prevent graft-versus-host disease (GVHD) after related donor allogeneic hematopoietic cell transplantation (HCT). Biol Blood Marrow Transplant. 2008;14:1017-1021.

36. Nasilowska-Adamska B, Rzepecki P, Manko J, et al. The influence of palifermin (Kepivance) on oral mucoasitis and acute graft versus host disease in patients with hematological diseases undergoing hematopoietic stem cell transplant. Bone Marrow Transplant. 2007;40:983-938.

37. Alderson R, Gohari-Fritsch S, Olsen H, Roschke V, Vance C, Connolly $\mathrm{P}$. In vitro and in vivo effects of repifermin (keratinocyte growth factor-2, KGF-2) on human carcinoma cells. Cancer Chemother Pharmacol. 2002;50:202-212.

38. Freytes CO, Ratanatharathorn V, Taylor C, et al. Phase I/II randomized trial evaluating the safety and clinical effects of repifermin administered to reduce mucositis in patients undergoing autologous hematopoietic stem cell transplantation. Clin Cancer Res. 2004;10:8318-8324.

39. Alvarez E Fey EG, Valax P, et al. Preclinical characterization of CG53135 (FGF-20) in radiation and concomitant chemotherapy/radiation-induced oral mucositis. Clin Cancer Res. 2003;9:3454-3461.

40. Alvarez E, Gerlach VL, Gerwien RW, et al. single-dose prevention or short-term treatment with fibroblast growth factor-20 (CG53135-05) reduces the severity and duration of oral mucositis. Support Cancer Ther. 2005;2:122-127.

41. Shuster MW, Shore TB, Harpel JG, et al. Safety and tolerability of velafermin (CG53135-05) in patients receiving high-dose chemotherapy and autologous peripheral blood stem cell transplant. Support Care Cancer. 2008;16:477-483. 